\title{
Absolute Stability Analysis of Sampled-Data Scaled Bilateral Teleoperation Systems
}

\author{
Ali Jazayeri and Mahdi Tavakoli \\ Department of Electrical and Computer Engineering, University of Alberta, Edmonton, AB T6G 2V4, Canada \\ Email: ali.jazayeri@ualberta.ca, tavakoli@ece.ualberta.ca
}

\begin{abstract}
Stability of a bilateral teleoperation system may be jeopardized by controller discretization, which has been shown to involve energy leaks. This paper proposes a novel approach to analyzing the absolute stability of sampled-data bilateral teleoperation systems consisting of discrete-time controllers and continuous-time master, slave, operator, and environment. The proposed stability analysis permits scaling and delay in the master and the slave positions and forces. The absolute stability conditions reported here impose bounds on the gains of the discrete-time controller, the damping terms of the master and the slave, and the sampling time. A design-related application of these results is in proper selection of various control parameters and the sampling rate for stable teleoperation under discrete-time control. To explore the trade-off between the control gains and the sampling time, it is studied how large sampling times, which require low control gains for maintaining stability, can lead to unacceptable teleoperation transparency and human task performance in a teleoperated switching task. This shows that the effect of sampling time must be taken into account because neglecting it (as in the absolute stability literature) undermines both stability and transparency of teleoperation. The resulting absolute stability condition has been verified via experiments with two Phantom Omni robots.
\end{abstract}

\section{INTRODUCTION}

A teleoperation system consists of a user interface ("master") that interacts with a human operator and a remote robot ("slave") that interacts with an environment. In bilateral teleoperation, while the slave robot mimics the motions of the master robot, the contact forces between the slave and the environment must be displayed to the operator by the master. Bilateral teleoperation has applications including telesurgery and remote underwater or space exploration. Surveys on bilateral teleoperation can be found in (Hokayem and Spong, 2006; Arcara and Melchiorri, 2002).

Teleoperation controllers are designed to meet two fundamental objectives, namely stability and transparency. Transparency is defined as matching of positions and forces of the master and the slave, and ensures that the environment's impedance is transmitted to the operator with no distortion. There is a trade-off between transparency and stability of a teleoperation system - the best performance is achieved by the least conservative stabilizing controller (Lawrence, 1993; Aziminejad et al., 2008).

\section{A. Teleoperation stability analysis}

To analyze the closed-loop stability of a teleoperation system, one can assume that the operator and the environment models are known, e.g. in (Tavakoli et al., 2008). While this assumption will simplify the stability analysis, it cannot be made in practice because the dynamic parameters of the human operator change in response to the specific requirements of the task at hand (Matsuoka and Howe, 2000), and the dynamic parameters of the environment are uncertain, time-varying and nonlinear.

Modeling the teleoperation system as a two-port network (teleoperator comprising the master, the controller and communication channel, and the slave) coupled to two one-port networks (environment and operator) paved the way for ensuring closed-loop stability via teleoperator passivity, i.e., ensuring that the two-port network teleoperator is passive, which physically means the teleoperator is not generating any energy (Anderson and Spong, 1989; Nuno et al., 2011). Indeed, ensuring the passivity of the teleoperator along with the assumed passivity of its two terminations will guarantee the passivity of the resulting interconnection and thus the closed-loop stability of the teleoperation system (Hannaford, 1989). The human operator impedance has been argued to be passive (Hogan, 1989). Passivity of the two-port teleoperator can be investigated via the scattering framework or Raisbeck's criterion (Haykin, 1970; Mendez and Tavakoli, 2010). In another passivity approach known as time domain passivity observer / passivity controller, the teleoperator's passivity is monitored in real time and in case of non-passivity the system is passified by adding damping to the system (Ryu et al., 2004).

Absolute stability analysis relaxes the passivity assumption on the teleoperator meaning that it allows the teleoperator to be non-passive as long as the closed-loop stability of the teleoperation system is preserved. Similar to passivity, the absolute stability approach assumes that the environment and the operator are passive but otherwise arbitrary. The absolute stability of a continuous-time two-port network can be assessed using Llewellyn's criterion (Mahvash and Okamura, 2007; Cho and Park, 2005; Abbott and Okamura, 2003). Recent studies have introduced geometric approaches inspired by criteria for unconditional stability of microwave systems to study the absolute stability of teleoperation systems (Haddadi and Hashtrudi-Zaad, 2009). 
The proposed method by Haddadi and Hashtrudi-zaad allows the environment and the operator to be non-passive with bounded impedance while the overall continuous-time teleoperation system is still stable.

Absolute stability breaks down the teleoperation system to three main blocks as shown in Fig. 1: A human operator (1-port network), an environment (1-port network), and a teleoperator (2-port network). The teleoperator absolute stability is concerned with stability of the overall teleoperation system having assumed that the two 1-port terminations are passive but otherwise arbitrary. As shown in Fig. 2, the absolute stability of a 2-port network is also equivalent to the passivity of the 1-port network resulting from connecting the other port of the 2-port network to a passive termination (Haddadi and Hashtrudi-Zaad, 2009). The challenge that is fully addressed in this paper is how to ensure stability of the overall teleoperation system when the only information about the terminations (i.e., the human operator and the environment) is their passivity. The assumption of termination passivity has been integrated into the stability analysis using the mapping of the positive real region to a unit disc in the Nyquist plane by finding the proper linear fractional transformation. A simpler case of such mapping has been first introduced by Colgate and Schenkel (Colgate and Schenkel, 1997) for a 1-port system (i.e. the virtual wall) and in this paper has been extended to 2-port network by solving the combined dynamics of both the master and the slave robots in a teleoperation system.

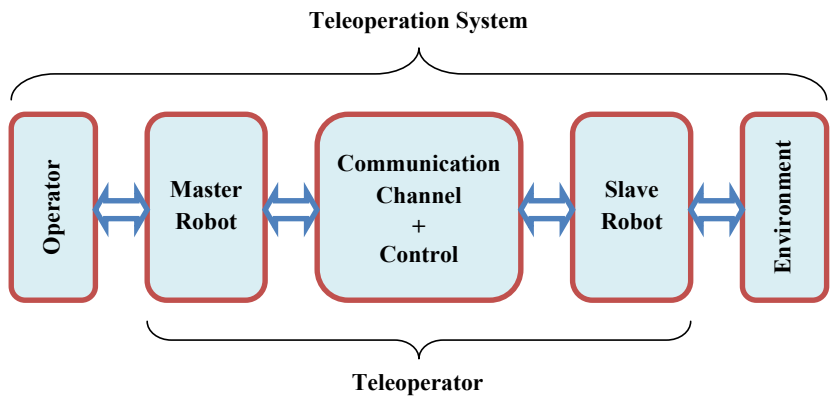

Fig. 1. The teleoperation system versus the teleoperator.

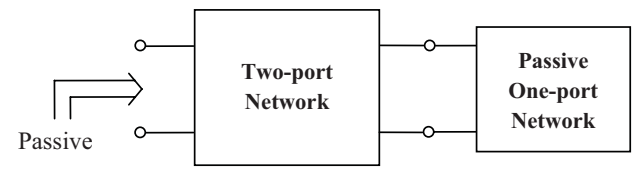

Fig. 2. Connecting a passive one-port network to any port of an absolutely stable two-port network results in a passive one-port network

As mentioned above, the assumption of passivity of the 1-port network terminations can be expressed by their positive realness for linear systems. A system with transfer function $Z(s)$ is positive real if and only if

1) $Z(s)$ has no pole in the right half plane (RHP).

2) Any poles of $Z(s)$ on the imaginary axis are simple with real and positive residues.

3) $\operatorname{Re}\{Z(s)\} \geq 0$ for all $\omega>0$.

The effect of position and/or force scaling on the stability and passivity of a teleoperation system is a nontrivial problem. The conventional two-port network passivity analysis cannot be directly used in a scaled teleoperation system with arbitrary position and force scaling factors for the following reason. The two-port network representing the teleoperator is passive if the work done by the operator and the environment on it is non-negative at all times and for all inputs and initial conditions:

$$
\int_{0}^{t} f_{h}(\tau) \dot{x}_{m}(\tau) d \tau+\int_{0}^{t} f_{e}(\tau) \dot{x}_{s}(\tau) d \tau>0
$$

where $f$ and $\dot{x}$ stand for force and velocity and subscript $h, m, e$ and $s$ correspond to hand, master, environment and slave, respectively. The above energy balance equation has relied on defining the power at the input and output ports as the multiplication of a velocity and a force. If $x_{m}$ and $x_{s}$ are scaled with respect to each other, then it is obvious that $f_{h}$ and $f_{e}$ need to be at the inverse scale to make the input and the output powers comparable in the conventional passivity definition of (1) see (Kosuge et al., 1996; Cho and Park, 2002). In other words, the conventional passivity definition (1) cannot hold if both velocity and force at one termination of a teleoperation are at a smaller scale than those at the other termination which is against the transparency requirement (Boukhnifer and Ferreira, 2006). On the other hand, it will be elaborated that the proposed absolute stability approach is able to tackle the stability of a scaled teleoperation system with the same ease as when there is no power scaling. 


\section{B. Sampled-data teleoperation}

A major challenge in stability analysis of teleoperation systems that is addressed in this paper is the effect of controller discretization. The discretization of a stabilizing continuous-time controller does not necessarily preserve the stability (Gillespie and Cutkosky, 1996; Leung and Francis, 1992). In fact, the stability of the closed-loop system will be degraded due to the energy-instilling behaviour of the Zero Order Hold (ZOH) (Gillespie and Cutkosky, 1996). Moreover, once the continuous-time controllers of a continuous-time system are substituted by their discrete-time counterparts, the resulting sampled-data system will perform poorly especially if the sampling time is comparable to the fast dynamics of the controlled system. Fast dynamics exist, for example, when the environment of a teleoperated robot is stiff resulting in high-frequency contact forces. Thus, a sampled-data system analysis is required to consider the impact of discretization in a bilateral teleoperation system (Sheng and Liu, 2004). Consider haptic teleoperation on a finite-impedance object where slave-environment interaction forces are measured by a force sensor, sampled and fed back to the user by a discrete-time controller. As the slave robot penetrates the environment, right at the edge of the environment, the environment does not resist and the force it applies to the slave robot is zero. As the slave robot's penetration into the environment increases, the resistive force coming from the environment increases. The measured contact force between the slave and the environment is sampled (during analog-to-digital conversion), and fed back to the human operator. Therefore, at any point in time during the penetration of the slave into the environment, the reflected force to the human operator corresponds to the last-sampled contact force, which has been due in response to a less deep penetration compared to the current penetration of the slave into the environment. Therefore, the reflected force to the human operator will be less than the actual slave/environment contact force. Conversely, when the slave moves out of the environment, the contact force decreases as time goes by. The sampled contact force, which is reflected to the human operator, will have a slight lag compared to the actual contact force, and hence will be larger than the actual contact force. Thus, the user's legitimate expectation that a passive environment would not generate energy is violated. Indeed, as the user utilizes the teleoperation system to probe the environment by pushing and letting go of the user interface, the energy-instilling sampled-data controller presents the environment to the user as one emitting energy and causing vibrations - an effect never observed when touching the same environment directly by hand. Such energy leaks have been investigated for haptic interaction with a virtual wall as will be discussed next, but not for haptic interaction with a physical environment via a computer-controlled teleoperation system for absolute stability analysis. A force reflective virtual reality system is similar to a bilateral teleoperation system in that the human operator feels discrete contact forces in each sampling time when applying position commands through the haptic user interface. For discrete-time haptic simulation of a virtual wall modeled by $B s+K$, where $B$ is the virtual stiffness and $K$ is the virtual damping, assuming that the haptic interface damping is $b$ and the sampling time is $T$, the stability condition has been found to be $b>K T / 2-B$ (Gil et al., 2004). This is clearly less conservative than the passivity condition for the same system, which is $b>K T / 2+B$ (Colgate and Schenkel, 1997). While the effect of discretization-induced energy leaks caused by the sampled-data nature of the system has been investigated for haptic interaction with a virtual wall, e.g. (Diolaiti et al., 2006), it has not been studied in the context of bilateral teleoperation where the slave interacts with a physical environment. Investigating the latter is the contribution of this paper.

Past papers dealing with the effect of discretization have either modified the teleoperation controllers to ensure the sampleddata system stability, or have analyzed the stability of the sampled-data system with discretized counterparts of the original continuous-time controllers (Sheng and Liu, 2004). In one of the first researches toward the controller design for a sampleddata teleoperation system, six low-pass filters were added to the control structure to stabilize the overall teleoperation system, which resulted in its sluggish performance (Leung and Francis, 1992). It was shown that for step-invariant discretization of the sampled-data teleoperation system, there exists an upper bound on the sampling time to keep the system stable. As for stability analysis of the sampled-data teleoperation system, the research is focused either on the absolute stability of the discrete-time communication channel (Berestesky et al., 2004) or the stability of the overall teleoperation system for known models of the environment (Aziminejad et al., 2008). Other approaches for sampled-data teleoperation system consider passivity to ensure the stability of the teleoperator (Secchi et al., 2007).

\section{Sampled-data teleoperation stability analysis}

Knowing the bounds on the parameters of the teleoperator model and the controller for ensuring absolute stability provides guidelines for designing controllers with high gains as needed for transparent teleoperation - it will be shown in this paper that increasing the control gains beyond a limit jeopardizes stability. In addition, certain applications such as texture recognition require high-frequency force feedback, increasing the demand for transparency over high frequencies and shrinking the stability margin. In the controller design for such a system, knowing the boundaries between the stable and unstable regions will be very useful. Another reason to know the bounds on the system parameters is that, as it will be shown later, there is a tradeoff between the sampling time and the maximum environment stiffness for stable teleoperation. When the slave robot is in hard contact (i.e., the environment stiffness is very large), the system requires very small sampling times, which exceeds the physical constraints of the discrete-time control hardware. Knowing the minimum sampling time helps to determine the maximum environment stiffness for which the teleoperator will be absolutely stable. Lastly, the absolute stability condition becomes particularly important with robots that, for performance reasons, have been designed to have low dampings (e.g., by 
minimizing friction). As shown in this paper, the stability condition puts a lower bound on the robot damping. Therefore, with a fixed robot damping, the controller is designed according to the absolute stability condition to ensure that the teleoperation system remains absolutely stable.

In this paper, a new absolute stability condition is developed for a sampled-data teleoperator without assuming any model for the operator or the environment as long as they are passive. The effect of the zero order hold is considered in the absolute stability analysis of the system. The absolute stability permits the teleoperator to be passive or nonpassive, resulting in a less conservative condition compared to the teleoperator passivity condition, thus allowing for higher teleoperation transparency and inclusion of arbitrary position and force scaling factors between the master and the slave. Unlike most of absolute stability methods in the literature, this new analysis considers continuous-time robots working with discrete-time controllers with arbitrary scaling factors for position and force. The condition can be used as a guideline for designing stabilizing and high-transparency controllers for sampled-data teleoperation systems.

This paper is organized as follows. A sampled-data bilateral teleoperation system is modeled in Section II. This model is later used in Section III to derive a condition for absolute stability of the sampled-data teleoperator. A few special cases are considered in Section IV to result in more practical conditions. Then, the experimental results on a pair of Phantom Omni robots are presented in Section V followed by the experimental results on a three-way slide switch in Section VI. Lastly, concluding remarks are given in Section VII.

\section{Modelling of SAmpled-Data Teleoperation Systems}

To study absolute stability, the dynamic models of the master robot and the slave robot are required. The dynamics of the master and slave robots are

$$
\begin{aligned}
-f_{m}+f_{h} & =m_{m} \ddot{x}_{m}+b_{m} \dot{x}_{m} \\
-f_{s}+f_{e} & =m_{s} \ddot{x}_{s}+b_{s} \dot{x}_{s}
\end{aligned}
$$

where $f_{h}$ is the interaction force between the master and the operator's hand, $m_{m}$ and $b_{m}$ are the master robot's inertia and dampings, respectively, and $x_{m}$ and $f_{m}$ are the position and actuator force of the master robot. Also, $f_{e}, m_{s}, b_{s}, x_{s}$ and $f_{s}$ are the slave and the environment counterparts. In the $s$-domain, (2) can be rewritten as

$$
\begin{aligned}
s X_{m} & =\frac{1}{m_{m} s+b_{m}}\left(-F_{m}+F_{h}\right) \\
s X_{s} & =\frac{1}{m_{s} s+b_{s}}\left(-F_{s}+F_{e}\right)
\end{aligned}
$$

The operator and the environment can be modeled as

$$
\begin{aligned}
\tilde{F}_{h}-F_{h} & =Z_{h}(s) s X_{m} \\
\tilde{F}_{e}-F_{e} & =Z_{e}(s) s X_{s}
\end{aligned}
$$

where $\tilde{F}_{h}$ and $\tilde{F}_{e}$ are the exogenous hand and environment forces, respectively. Also, $Z_{h}$ and $Z_{e}$ are the operator's hand and environment impedances, respectively.

Fig. 3 shows the sampled-data bilateral teleoperation system block diagram. To discretize the continuous-time signals, they are sampled at time instants separated by $T$ (Ogata, 1995). The ideal sampler unit generates the output $x^{*}(t)$ from the input $x(t)$ where

$$
x^{*}(t)=\sum_{k=0}^{\infty} x(k T) \delta(t-k T)
$$

The Laplace transform of the sampled signal is given by

$$
X^{*}(s)=\mathcal{L}\left\{x^{*}(t)\right\}=\sum_{k=0}^{\infty} x(k T) e^{-k T}
$$

The $z$-transform of the sampled signal is given by

$$
X(z)=\mathcal{Z}\left\{x^{*}(t)\right\}=\left.X^{*}(s)\right|_{s=1 / T \ln z}
$$

The master and the slave position output signals $\left(x_{m}\right.$ and $\left.x_{s}\right)$ are converted to discrete-time $\left(x_{m}^{*}\right.$ and $\left.x_{s}^{*}\right)$ using sampler blocks and fed to the controllers. The controllers deal only with discrete-time signals at their input and output. The digital controller 


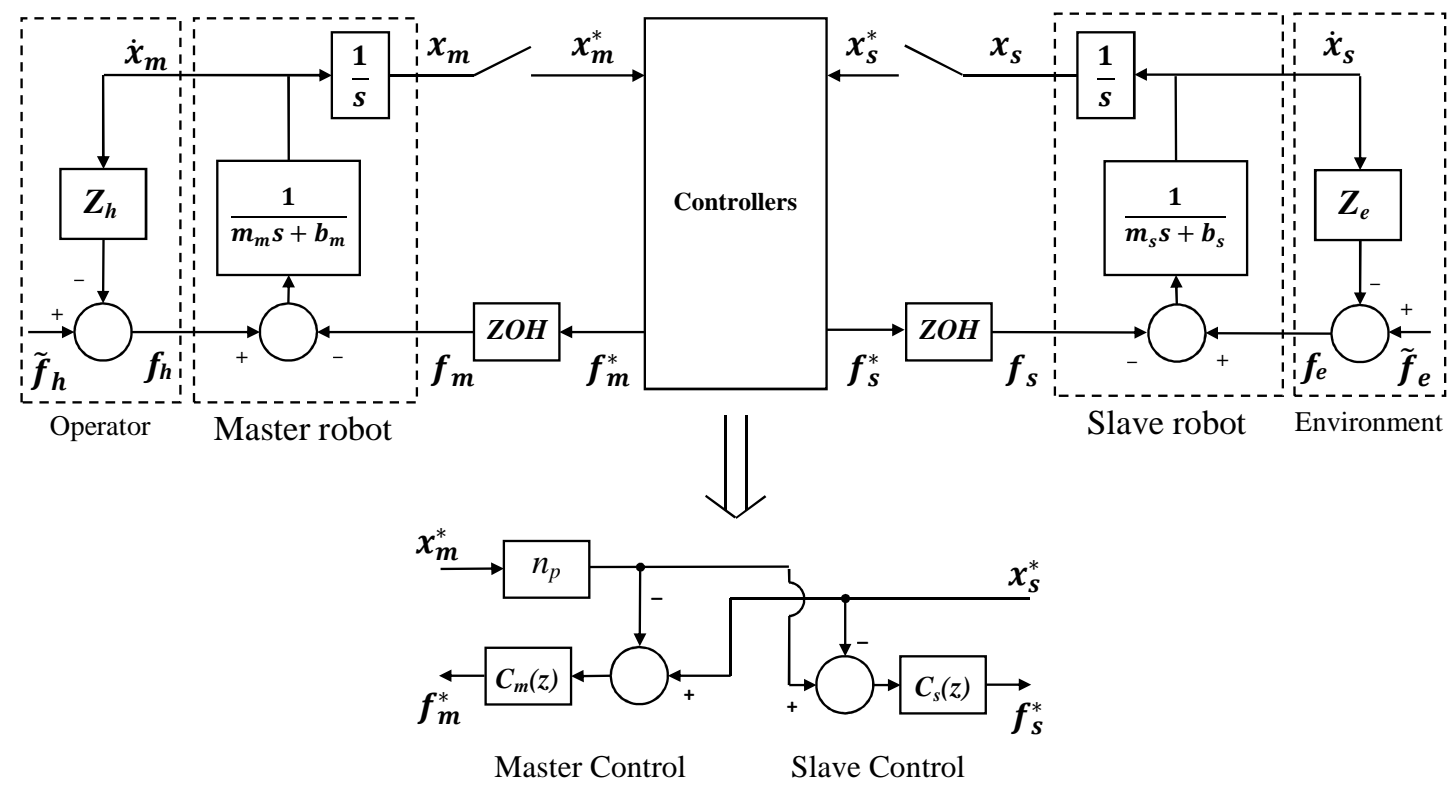

Fig. 3. Discrete-time controlled bilateral teleoperation system

outputs $\left(f_{m}^{*}\right.$ and $\left.f_{s}^{*}\right)$ are converted to continuous-time $\left(f_{m}\right.$ and $\left.f_{s}\right)$ using Zero-Order-Hold (ZOH) blocks with the transfer function $G_{h}(s)=\left(1-e^{-s T}\right) / s T$ :

$$
\begin{aligned}
F_{m}(s) & =\frac{1-e^{-s T}}{s T} F_{m}^{*}(s) \\
F_{s}(s) & =\frac{1-e^{-s T}}{s T} F_{s}^{*}(s)
\end{aligned}
$$

Assume that the teleoperation system uses a position-error-based (PEB) controller. A PEB controller is chosen because, with a direct force reflection (DFR) controller, even the continuous-time teleoperation system will not be absolutely stable (Appendix A). As depicted in Fig. 3, the PEB controllers work based on the position error between the master and the slave. The outputs of the master and slave controllers $F_{m}^{*}$ and $F_{s}^{*}$ will be sampled-data signals

$$
\begin{aligned}
F_{m}^{*}(s) & =C_{m}(z)\left[X_{s}^{*}(s)-n_{p} X_{m}^{*}(s)\right] \\
F_{s}^{*}(s) & =C_{s}(z)\left[n_{p} X_{m}^{*}(s)-X_{s}^{*}(s)\right]
\end{aligned}
$$

where $n_{p}$ is the scaling factor between the master and the slave positions. Similar to the unity-scale teleoperation (Jazayeri and Tavakoli, 2010), as the controller drives the position error $e=x_{s}-n_{p} x_{m}$ to zero, the master and slave positions will have the following ratio

$$
\frac{x_{s}}{x_{m}}=n_{p}
$$

Here, the ${ }^{*}$ superscript requires us to use the infinite impulse-train definition (5) of the sampler in the derivations that follow.

In order to be able to derive the closed-loop transfer function, from Fig. 3, the continuous-time transfer functions from $f_{m}$ to $x_{m}$ and from $f_{s}$ to $x_{s}$ can be found as

$$
\begin{gathered}
\frac{X_{m}}{F_{m}}=\frac{1}{s} \cdot \frac{\frac{1}{m_{m} s+b_{m}}}{1+\frac{Z_{h}}{m_{m} s+b_{m}}}=\frac{1}{s} \cdot \frac{1}{m_{m} s+b_{m}+Z_{h}} \\
\frac{X_{s}}{F_{s}}=\frac{1}{s} \cdot \frac{\frac{1}{m_{s} s+b_{s}}}{1+\frac{Z_{e}}{m_{s} s+b_{s}}}=\frac{1}{s} \cdot \frac{1}{m_{s} s+b_{s}+Z_{e}}
\end{gathered}
$$

Note that the only knowledge about the transfer functions of the human operator $Z_{h}$ and the environment $Z_{e}$ is that they are passive. When the above continuous transfer functions are combined with the models of the two zero-order-holds in (8), the transfer functions from $f_{m}^{*}$ to $x_{m}$ and from $f_{s}^{*}$ to $x_{s}$ can be found as

$$
\begin{aligned}
G_{m}(s) & =\frac{X_{m}(s)}{F_{m}^{*}}=\frac{1}{m_{m} s+b_{m}+Z_{h}(s)} \cdot \frac{1-e^{-s T}}{s T} \cdot \frac{1}{s} \\
G_{s}(s) & =\frac{X_{s}(s)}{F_{s}^{*}}=\frac{1}{m_{s} s+b_{s}+Z_{e}(s)} \cdot \frac{1-e^{-s T}}{s T} \cdot \frac{1}{s}
\end{aligned}
$$


By substituting these into the model of the sampler (5), the overall system equations (9) become

$$
\begin{aligned}
F_{m}^{*}(s) & =C_{m}\left(e^{s T}\right)\left[-n_{p} G_{m}^{*}(s) F_{m}^{*}(s)+G_{s}^{*}(s) F_{s}^{*}(s)\right] \\
F_{s}^{*}(s) & =C_{s}\left(e^{s T}\right)\left[-G_{s}^{*}(s) F_{m}^{*}(s)+n_{p} G_{m}^{*}(s) F_{s}^{*}(s)\right]
\end{aligned}
$$

where $G_{m}^{*}(s)$ and $G_{s}^{*}(s)$ are the following discrete-time transfer functions:

$$
\begin{aligned}
G_{m}^{*}(s) & =\frac{1}{T} \sum_{k} G_{m}\left(s+j k \omega_{T}\right) \\
G_{s}^{*}(s) & =\frac{1}{T} \sum_{k} G_{s}\left(s+j k \omega_{T}\right)
\end{aligned}
$$

And $\omega_{T}=2 \pi / T$ is the sampling frequency.

\section{MAIN RESULT}

In section II, the sampled-data teleoperation system was modelled and resulted in closed-loop equations (12). Our main theorem for testing absolute stability of the sampled-data teleoperation system will be given after the following definition and lemma.

Definition 1. A teleoperator is called absolutely (or unconditionally) stable when coupling it to any passive but otherwise arbitrary environment and operator terminations results in a stable teleoperation system ${ }^{1}$. Otherwise, the teleoperation system is potentially unstable, which does not necessarily mean instability.

Lemma 1. The closed-loop characteristic equation of the sampled-data teleoperation system in Fig. 3 is

$$
1+n_{p} C_{m}\left(e^{s T}\right) G_{m}^{*}(s)+C_{s}\left(e^{s T}\right) G_{s}^{*}(s)=0
$$

Proof: It is easy to see that the transfer function from $\tilde{F}_{h}$ to $X_{m}$ is

$$
\frac{X_{m}(s)}{\tilde{F}_{h}(s)}=\frac{\left(1+C_{s}\left(e^{s T}\right) G_{s}^{*}(s)\right) H_{m}(s)}{1+n_{p} C_{m}\left(e^{s T}\right) G_{m}^{*}(s)+C_{s}\left(e^{s T}\right) G_{s}^{*}(s)}
$$

where

$$
H_{m}(s)=\frac{1}{Z_{m}(s)+Z_{h}(s)} \cdot \frac{1}{s}
$$

and the master robot impedance is defined by $Z_{m}=m_{s} s+b_{m}$. It can be shown that (14) is the also denominator of all transfer functions from inputs $\tilde{F}_{h}$ or $\tilde{F}_{e}$ to any other output.

The open-loop system equations (12) can be written as

$$
\left[\begin{array}{cc}
1+n_{p} C_{m} G_{m}^{*} & -C_{m} G_{s}^{*} \\
-n_{p} C_{s} G_{m}^{*} & 1+C_{s} G_{s}^{*}
\end{array}\right]\left[\begin{array}{c}
F_{m}^{*} \\
F_{s}^{*}
\end{array}\right]=\left[\begin{array}{l}
0 \\
0
\end{array}\right]
$$

The determinant of the above matrix gives the characteristic equation of the system to be (14).

Theorem 1. The sampled-data teleoperator resulting from using the discrete-time control laws (9) with the continuous-time system (2) as in Fig. 3 will be absolutely stable if

$$
\left\|M_{m} N_{m}+M_{s} N_{s}\right\|_{\infty}<1
$$

where $M_{m}, M_{s}, N_{m}$ and $N_{s}$ are linear fractional transformations defined as

$$
\begin{array}{ll}
N_{m}\left\{s, C_{m}\left(e^{s T}\right)\right\}= & \frac{n_{p} b_{s} C_{m}\left(e^{s T}\right) r(s)}{2 b_{m} b_{s}+n_{p} b_{s} C_{m}\left(e^{s T}\right) r(s)+b_{m} C_{s}\left(e^{s T}\right) r(s)} \\
N_{s}\left\{s, C_{s}\left(e^{s T}\right)\right\}= & \frac{b_{m} C_{s}\left(e^{s T}\right) r(s)}{2 b_{m} b_{s}+n_{p} b_{s} C_{m}\left(e^{s T}\right) r(s)+b_{m} C_{s}\left(e^{s T}\right) r(s)}
\end{array}
$$

\footnotetext{
${ }^{1}$ Stability of the teleoperation system is defined as $L_{2}$-stability of the system when the system input is the exogenous human operator force $\tilde{f}_{h}$ and the system output is the slave position $x_{m}$.
} 


$$
\begin{aligned}
M_{m}\left\{s, G_{m}^{*}\right\} & =-1+\frac{2 b_{m}}{r(s)} G_{m}^{*}(s) \\
M_{s}\left\{s, G_{s}^{*}\right\} & =-1+\frac{2 b_{s}}{r(s)} G_{s}^{*}(s)
\end{aligned}
$$

and

$$
r(j \omega)=\frac{T}{2} \frac{e^{-j \omega T}-1}{1-\cos \omega T}
$$

Proof:

For the sampled-data teleoperator's absolute stability, it is necessary and sufficient that the closed-loop characteristic equation (14) of the teleoperation system has all of its roots in the left half of the complex plane. The definition of absolute stability of a teleoperator assumes that the environment and the operator are passive and, therefore, their impedances are positive real functions. This is what the proof starts with.

In the Nyquist plane, $Z_{h}$ and $Z_{e}$ cover the entire right half plane due to their positive realness. Therefore, given that the master and slave robots have positive mass and damping, it is concluded that

$$
\begin{aligned}
1 /\left(m_{m} s+b_{m}+Z_{h}(s)\right) & \in \mathfrak{D}\left\{\frac{1}{2 b_{m}}, \frac{1}{b_{m}}\right\} \doteq \mathbb{D}_{m} \\
1 /\left(m_{s} s+b_{s}+Z_{e}(s)\right) & \in \mathfrak{D}\left\{\frac{1}{2 b_{s}}, \frac{1}{b_{s}}\right\} \doteq \mathbb{D}_{s}
\end{aligned}
$$

where $\mathfrak{D}\left\{x_{1}, x_{2}\right\}$ is a disk in the Nyquist plane with the center point of $\left(x_{1}, 0\right)$ and the diameter of $x_{2}$. The mappings in (19) are frequency-independent. Now, (19) can be replaced in (11) to ultimately determine the regions of $G_{m}^{*}$ and $G_{s}^{*}$ in (13). Because $\mathbb{D}_{m}$ and $\mathbb{D}_{s}$ are frequency-independent, they can be moved out of the summations in (13). The regions of $G_{m}^{*}$ and $G_{s}^{*}$ are, therefore, found as

$$
\begin{aligned}
G_{m}^{*} & \in r(j \omega) \mathbb{D}_{m} \\
G_{s}^{*} & \in r(j \omega) \mathbb{D}_{s}
\end{aligned}
$$

where $r(j \omega)$ is the frequency-dependent part in the summation (13) and can be calculated as

$$
r(j \omega)=\frac{1}{T} \sum_{k=-\infty}^{+\infty} \frac{1-e^{-\left(j \omega+j k \omega_{T}\right) T}}{\left(j \omega+j k \omega_{T}\right)^{2}}
$$

Then, (21) will yield (18).

Consequently, the regions covered by $G_{m}^{*}$ and $G_{s}^{*}$ consist of a frequency-dependent part $r(j \omega)$ as in (18) and a frequencyindependent part as in (19) that is shifted and scaled in the Nyquist plane. The areas in (20) can be mapped to the stable unit disc centered at the origin via the following transformations:

$$
\begin{aligned}
-1+\frac{2 b_{m}}{r(j \omega)} G_{m}^{*} & \in \mathfrak{D}\{0,2\} \\
-1+\frac{2 b_{s}}{r(j \omega)} G_{s}^{*} & \in \mathfrak{D}\{0,2\}
\end{aligned}
$$

Based on (22), the linear fractional transformations (LFT) defined by $M_{m}$ and $M_{s}$ in (17) map the regions of $G_{m}^{*}$ and $G_{s}^{*}$ in (20) to two unit discs.

Now, transformations $N_{m}$ and $N_{s}$ can be found such that the transformed characteristic equation

$$
1+M_{m} N_{m}+M_{s} N_{s}=0
$$

has the same roots as the original characteristic equation (14). To this end, replacing $M_{m}$ and $M_{s}$ from (17) into (23) leads us to the condition

$$
\begin{array}{r}
1+\frac{-r(s)+2 b_{m} G_{m}^{*}}{r(s)} N_{m}\left\{s, C_{m}\left(e^{s T}\right)\right\} \\
+\frac{-r(s)+2 b_{s} G_{s}^{*}}{r(s)} N_{s}\left\{s, C_{s}\left(e^{s T}\right)\right\} \\
=\kappa\left(1+n_{p} C_{m} G_{m}^{*}+C_{s} G_{s}^{*}\right)
\end{array}
$$

Note that (24) should be valid for any $G_{m}^{*}$ and $G_{s}^{*}$ and the coefficient $\kappa$ should be independent of $G_{m}^{*}$ and $G_{s}^{*}$. By solving (24), the transformations $N_{m}$ and $N_{s}$ can be found as in (16). Finally, the small gain theorem provides a sufficient condition for the stability of the characteristic equation (23) as given by (15) (van der Schaft, 1999). 
It is to note that the although (23) and (14) have similar form, they have very different interpretation and applying the small gain theorem on (14) gives an involve condition particularly due to the fact that the assumption on positive realness of the terminations will not be used and the it has the unknown models of the environment and the operator are still in the condition.

In Theorem 1, transformations $M_{m}$ and $M_{s}$ are unit discs in the Nyquist plane. Condition (15) is the general condition that the controllers $C_{m}$ and $C_{s}$ should meet to ensure that the sampled-data teleoperator is absolutely stable.

To achieve condition (15), a sufficient condition is

$$
\left\|M_{m} N_{m}\right\|_{\infty}+\left\|M_{s} N_{s}\right\|_{\infty}<1
$$

Since $M_{m}$ and $M_{s}$ are unit discs in the Nyquist plane, this sufficient condition for absolute stability reduces to

$$
\left\|N_{m}\right\|_{\infty}+\left\|N_{s}\right\|_{\infty}<1
$$

By substituting the definitions of $N_{m}$ and $N_{s}$ from (16) in the above, the sufficient condition for teleoperator absolute stability becomes

$$
\frac{\left|n_{p} b_{s} C_{m}\left(e^{j \omega T}\right) r(j \omega)\right|+\left|b_{m} C_{s}\left(e^{j \omega T}\right) r(j \omega)\right|}{\left|2 b_{m} b_{s}+b_{s} n_{p} C_{m}\left(e^{j \omega T}\right) r(j \omega)+b_{m} C_{s}\left(e^{j \omega T}\right) r(j \omega)\right|}<1
$$

where $r(j \omega)$ is defined in (18). Note that (27) gives an absolute stability condition that is not tied to any particular controller as long as the teleoperation system complies with PEB control architecture. For known models of the master and the slave robots, any given discrete-time controller can be tested using condition (27) to investigate the absolute stability of the sampled-data teleoperator. Also, note that although absolute stability is less conservative than passivity, some new conservativeness have been introduced by finding the stability condition using the small gain theorem.

Next, the communication channel is assumed to have constant delays in transmitting the signals and the absolute stability condition for such case has been derived as follows. The delay for the master side and the slave side are assumed to be independent and potentially different. Without loss of generality it is assumed that the position scaling is unity $n_{p}=1$. Furthermore, it is assumed that the delays in the communication channel are integer multiple of the sampling time of the controllers.

Theorem 2. For a position-error-based sample-data teleoperation system with the continuous-time open-loop dynamics (2), if there is a delay $T_{1}$ in communication path from the master to the slave and a delay $T_{2}$ in the opposite direction, the absolute stability condition is

$$
\frac{\left|D+b_{s} C_{m} r\right|+\left|D+b_{m} C_{s} r\right|+|D|}{\left|2 b_{m} b_{s} C_{m} C_{s}+b_{s} C_{m}^{2} C_{s} r+b_{m} C_{s}^{2} C_{m} r+D\right|}<1
$$

where

$$
D=\frac{r^{2}\left(1-e^{-\left(T_{1}+T_{2}\right) s}\right)}{2}
$$

Proof:

Substituting the controller laws for the master and the slave into the open-loop dynamics provides the following closed-loop dynamics for the time-delay sampled-data teleoperation system:

$$
\begin{aligned}
F_{m}^{*}(s) & =C_{m}\left(e^{s T}\right)\left[-G_{m}^{*}(s) F_{m}^{*}(s)+e^{-s T_{2}} G_{s}^{*}(s) F_{s}^{*}(s)\right] \\
F_{s}^{*}(s) & =C_{s}\left(e^{s T}\right)\left[-e^{-s T_{1}} G_{s}^{*}(s) F_{m}^{*}(s)+n_{p} G_{m}^{*}(s) F_{s}^{*}(s)\right]
\end{aligned}
$$

Compared to (12) The two new terms $e^{-s T_{1}}$ and $e^{-s T_{2}}$ are due to the time delay in the communication channel. For the sake of brevity, in the rest of the proof, the arguments $(s)$ and $\left(e^{s T}\right)$ of the transfer functions are omitted. Regrouping the controller outputs, the closed-loop equations can be written as

$$
\left[\begin{array}{cc}
1+C_{m} G_{m}^{*} & -e^{-s T_{2}} C_{m} G_{s}^{*} \\
-e^{-s T_{1}} C_{s} G_{m}^{*} & 1+C_{s} G_{s}^{*}
\end{array}\right]\left[\begin{array}{c}
F_{m}^{*} \\
F_{s}^{*}
\end{array}\right]=\left[\begin{array}{l}
0 \\
0
\end{array}\right]
$$

Similar to the delay-free derivation in (19) to (23), the absolute stability condition becomes

$$
\left\|M_{m} N_{m}+M_{s} N_{s}+M_{m} M_{s} N_{d}\right\|_{\infty}<1
$$

where

$$
N_{m}=\quad \frac{r^{2}\left(1-e^{-\left(T_{1}+T_{2}\right) s}\right)+2 C_{m}^{2} C_{s} r b_{s}}{4 b_{m} b_{s} C_{m} C_{s}+2 b_{s} r C_{m}^{2} C_{s}+2 b_{m} r C_{s}^{2} C_{m}+r^{2}\left[1-e^{\left.-\left(T_{1}+T_{2}\right) s\right]}\right.}
$$




$$
\begin{aligned}
& N_{s}=\quad \frac{r^{2}\left(1-e^{-\left(T_{1}+T_{2}\right) s}\right)+2 C_{s}^{2} C_{m} r b_{m}}{4 b_{m} b_{s} C_{m} C_{s}+2 b_{s} r C_{m}^{2} C_{s}+2 b_{m} r C_{s}^{2} C_{m}+r^{2}\left[1-e^{-\left(T_{1}+T_{2}\right) s}\right]} \\
& N_{d}= \\
& \frac{r^{2}\left(1-e^{-\left(T_{1}+T_{2}\right) s}\right)}{4 b_{m} b_{s} C_{m} C_{s}+2 b_{s} r C_{m}^{2} C_{s}+2 b_{m} r C_{s}^{2} C_{m}+r^{2}\left[1-e^{-\left(T_{1}+T_{2}\right) s}\right]}
\end{aligned}
$$

Also, $M_{m}$ and $M_{s}$ in (32) are the same unit discs defined in (17). Thus, a sufficient condition for absolute stability is

$$
\left\|N_{m}\right\|_{\infty}+\left\|N_{s}\right\|_{\infty}+\left\|N_{d}\right\|_{\infty}<1
$$

Substituting the definitions of $N_{m}, N_{s}$ and $N_{d}$ from (33)-(35) in (36), the absolute stability condition, which is the delayed counterpart of (27), becomes what is shown in (28).

\section{Special Cases}

Although condition (27) covers all controllers used in the PEB architecture of (9), verifying it can be difficult in the general case for arbitrarily controllers. In this section, certain assumptions on the controllers are shown to help to reduce the absolute stability condition (27) to useful bounds on the control parameters that make it easier to design stabilizing, high-performance controllers.

\section{A. Proportionally selected controllers}

Condition (27) includes both the master and the slave controllers, which can be arbitrarily selected. In practical design, an option is to select the controllers to be proportional to each other:

$$
C_{s}(z)=n_{c} C_{m}(z)
$$

where $n_{c}$ is an arbitrary positive constant. There is no restriction on the controllers $C_{s}(z)$ and $C_{m}(z)$.

Substituting (37) into (27) results in the following absolute stability condition:

$$
\frac{\left|n_{p} b_{s} C_{m}\left(e^{j \omega T}\right) r(j \omega)\right|+\left|n_{c} b_{m} C_{m}\left(e^{j \omega T}\right) r(j \omega)\right|}{\left|2 b_{m} b_{s}+b_{s} n_{p} C_{m}\left(e^{j \omega T}\right) r(j \omega)+n_{c} b_{m} C_{m}\left(e^{j \omega T}\right) r(j \omega)\right|}<1
$$

Replacing the complex term $C_{m}\left(e^{j \omega T}\right) r(j \omega)$ by $p+j q$ in (38) gives

$$
\frac{n_{p} b_{s} \sqrt{p^{2}+q^{2}}+n_{c} b_{m} \sqrt{p^{2}+q^{2}}}{\sqrt{\left(2 b_{m} b_{s}+n_{p} b_{s} p+n_{c} b_{m} p\right)^{2}+\left(n_{p} b_{s} q+n_{c} b_{m} q\right)^{2}}}<1
$$

which can be further manipulated to conclude the following absolute stability condition:

$$
\frac{b_{m} b_{s}}{n_{p} b_{s}+n_{c} b_{m}}>-\operatorname{Re}\left\{C_{m}\left(e^{j \omega T}\right) r(j \omega)\right\}
$$

It should be noted that in (40) the ratio of the master and the slave controllers $n_{c}$ is not necessarily related to the position scaling $n_{p}$. This means that (40) covers the most general case while certain applications may require specific relationships between the two.

For the sake of simplicity in the following discussion, let us assume that $n_{c}=n_{p}=1$. With this assumption, the absolute stability condition (40) becomes

$$
\frac{b_{m} b_{s}}{b_{m}+b_{s}}>-\operatorname{Re}\left\{C_{m}\left(e^{j \omega T}\right) r(j \omega)\right\}
$$

Let us compare the absolute stability condition (41) against the passivity condition for a similar sampled-data teleoperator (i.e., when there is no position or controller scaling). As shown in (Jazayeri and Tavakoli, 2011), the sampled-data teleoperator passivity conditions are $b_{m}>-2 p$ and $b_{s}>-2 p$ to be satisfied simultaneously. When the robot dampings are equal $\left(b_{m}=b_{s}\right)$, the absolute stability condition (41) reduces to $b_{m}=b_{s}>-2 p$, which is the same as the passivity condition. However, when the dampings are not equal $\left(b_{m} \neq b_{s}\right)$, the absolute stability condition (41) allows the dampings to vary as long as they satisfy $1 / b_{m}+1 / b_{s}<-1 / p$ whereas the passivity conditions require each of $1 / b_{m}$ and $1 / b_{s}$ to be less than $-1 /(2 p)$. In other words, the absolute stability condition is less conservative than the passivity condition under similar circumstances. For instance, as shown in Fig. 4, where the slave robot damping violates the passivity condition $b_{s}>-2 p$, the absolute stability condition (41) will be satisfied if the master robot has a high enough damping.

In another analysis on the absolute stability condition (40), consider a micro/macro teleoperation scenario where the slave robot is substantially heavier/larger than the master robot, e.g., the remote manipulation of an excavator robot by a lighter/smaller haptic device. The slave damping is much higher than the master damping $\left(b_{s} \gg b_{m}\right)$, and (40) can be approximated as $b_{m} / n_{p}>-p$. First, this new condition only puts a lower bound on the master damping as the slave damping is already large enough. Second, the condition will be relieved further if $n_{p}=x_{s} / x_{m}$ is large, which is indeed the case in the tele-excavation 


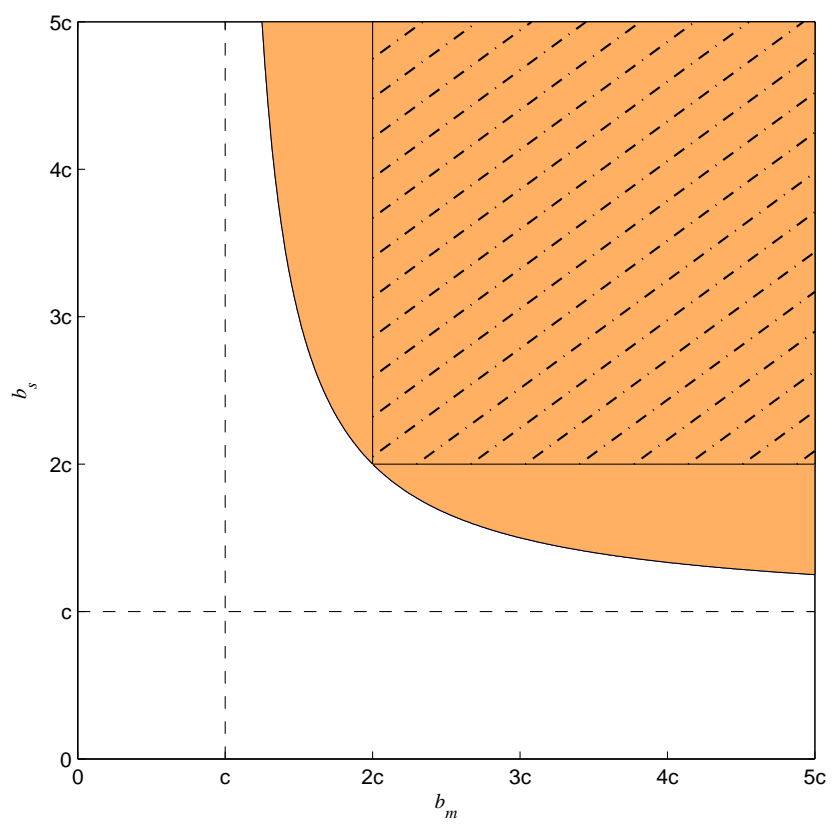

Fig. 4. Absolute stability and passivity regions in the plane of the master robot damping $b_{m}$ versus the slave robot damping $b_{s}$. Blue shows the absolute stability region and hatched corresponds to the passivity region and $c$ is the right hand side of (40).

application. Conversely, in macro/micro teleoperation, the slave robot has significantly smaller scale and weight than the master robot, implying that the passivity condition lower bounds the damping of the slave robot.

While the above were two illustrative examples, in general the absolute stability condition found in this paper presents improvements over the passivity condition. First, as described above through an illustrative example, the condition (40) allows the master and slave dampings to vary in a more flexible and less conservative manner. Second, the absolute stability condition (40) allows to include arbitrary position scaling $n_{p}$ (and controller scaling $n_{c}$ ) in the system that meet the practical requirements of the task at hand at no cost.

\section{B. PD controllers}

The absolute stability condition (40) is valid for all controllers in the position error based structure of (9). For a known controller structure, this condition can be further simplified. In the following, a continuous-time PD controller $C_{m}(s)=K+B s$ is discretized using the bilinear transformation method to $C_{m}(z)=K+B(z-1) / T z$ (Ogata, 1995). Substituting the PD controller in (40) yields the following absolute stability condition:

$$
\frac{b_{m} b_{s}}{n_{p} b_{s}+n_{c} b_{m}}>\frac{K T}{2}-B \cos \omega T
$$

Condition (42) depends on the frequency $\omega$. Since $\cos \omega T$ varies between -1 and 1, a sufficient condition for absolute stability of the teleoperator over all frequencies will be

$$
\frac{b_{m} b_{s}}{n_{p} b_{s}+n_{c} b_{m}}>\frac{K T}{2}+B
$$

The absolute stability condition (43) indicates that the higher the sampling time and the controller gains, the closer the system is to potential instability. In a practical teleoperation system, the robot dampings $b_{m}$ and $b_{s}$ are physical characteristics of the robots and are typically fixed. The controller scaling $n_{c}$, which in PEB control also reflects the force scaling, and the position scaling $n_{p}$ and are determined by the physical requirements of the teleoperation system and the task at hand. Typically, the sampling time $T$ is lower bounded as a result of the limited A/D conversion, D/A conversion, and computation speeds of the control hardware. Therefore, the parameters in (43) that can most easily be set are the PD controller gains.

On the other hand, as mentioned in Section I, besides stability the teleoperation control design has to strive for transparency. Higher transparency can be achieved by increasing the controller gains, as shown in the following Remark.

Remark 1. In a continuous-time PEB teleoperation system, increasing the controller gains will result in a higher teleoperation transparency. Indeed, if the continuous-time counterpart of Fig. 3 is modelled in the hybrid matrix form,

$$
\left[\begin{array}{c}
F_{m}(s) \\
-V_{s}(s)
\end{array}\right]=H(s)\left[\begin{array}{c}
V_{m}(s) \\
F_{s}(s)
\end{array}\right]
$$


the hybrid matrix $H(s)$ is

$$
H(s)=\left[\begin{array}{cc}
Z_{m}+C_{m} \frac{Z_{s}}{Z_{s}+C_{s}} & \frac{C_{m}}{Z_{s}+C_{s}} \\
-\frac{C_{s}}{Z_{s}+C_{s}} & \frac{1}{Z_{s}+C_{s}}
\end{array}\right]
$$

where $Z_{m}(s)=m_{m} s+b_{m}$ and $Z_{s}(s)=m_{s} s+b_{s}$. For perfect master/slave position and force matching, the transparent hybrid matrix must be

$$
H_{\text {ideal }}=\left[\begin{array}{cc}
0 & 1 \\
-1 & 0
\end{array}\right]
$$

Comparison of (45) and (46) leads to the fact that increasing the controller gains of $C_{m}$ and $C_{s}$ increases teleoperation transparency.

By analogy, in a sampled-data teleoperation system, higher gains will deliver higher transparency. On the other hand, the absolute stability condition (43) imposes upper bounds on the controller gains, indicating a tradeoff between transparency and absolute stability. In the next section, these boundaries are tested via experiments designed to get the highest transparency while remaining stable.

\section{Impact of time delay}

The absolute stability condition (28) for delayed teleoperation systems has been verified for a given teleoperation system. A nominal teleoperation system has been defined to have the system parameters $K=1000, B=0, T=1^{m s}$ and $b_{m}=$ $b_{s}=b=1$. A stability index has been defined as the left hand side of (28) minus one, which should be negative to ensure the absolute stability of the delayed-teleoperation system. The resulting stability index has been plotted against changes in the system parameters, e.g., the controller gain and the time delay. The results have been shown in Fig. 5 and 6.

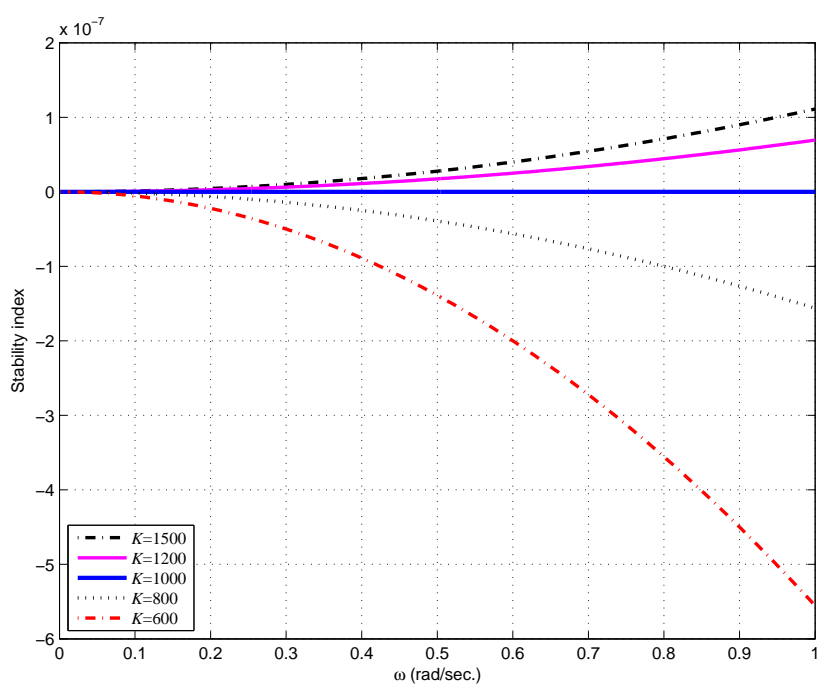

Fig. 5. Stability index vs. the controller gain when the delays $T_{1}=T_{2}=0$. As long as the controller gain $K$ satisfies (40), which is $K<1000$, the stability index remains negative (i.e., the teleoperation system remains absolutely stable).

As depicted in Fig. 5, by changing the controller gain in the absence of time delay, the absolute stability of the system is affected. A gain of $K=1000$ is the border line for stability; lower gains make the stability index negative and the teleoperation system absolutely stable. Fig. 6 shows that the delay can cause the stability index to become positive and consequently the teleoperation system not absolutely stable (i.e., potentially unstable). Furthermore, from Fig. 6 it is concluded that the absolute stability condition is violated for any non-zero delay.

\section{Experiments on a Scaled Dual Phantom Omni}

To verify the absolute stability condition, an experiment has been set up with two identical Phantom Omni robots from Sensable Technologies, Inc., which are 6-DOF haptic devices with 3 actuated and 3 free-running joints. Out of the three actuated joints of the robot, the first joint that rotates about the vertical is used in the experiment while the second and the third joints that form a parallel mechanism are in locked motion using high-gain controllers. As shown in Fig. 7, the operator interacts with the master robot while the slave robot is physically connected via a nonlinear spring to a stiff wall. For simplicity of the picture, in lieu of the physical wall and environment, symbols of the wall and the environment are displayed in Fig. 7.

The Phantom Omni robots are connected in daisy chain on a FireWire port and communicate with the same computer. The robots are interfaced with MATLAB Simulink@(from Mathworks, Natick, MA, USA) via a pair of QuARC $\mathbb{B}$ blocks 


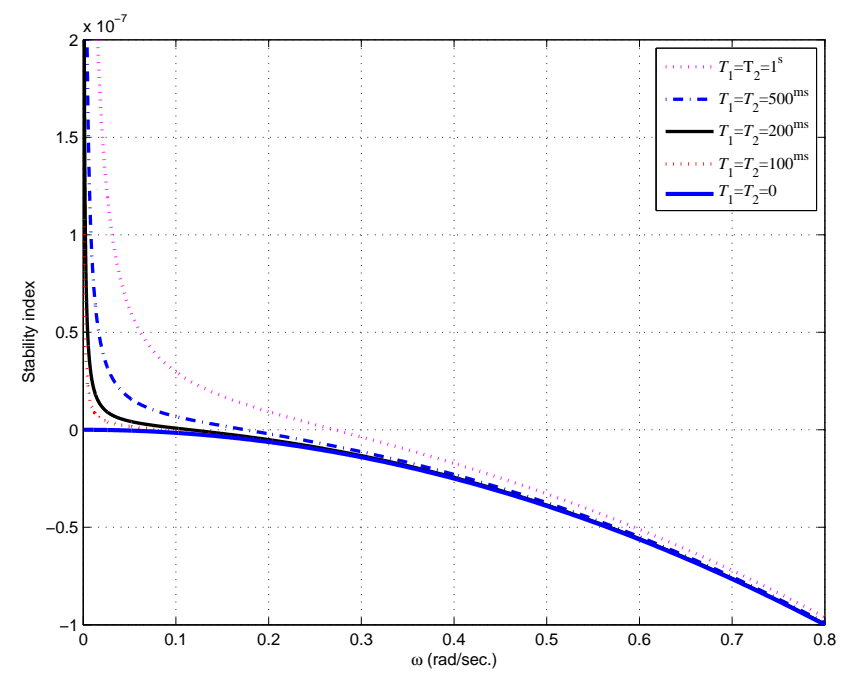

Fig. 6. Stability index vs. time delay when the controller gain $K=800$. As long as the delays satisfy (28), the stability index remains negative (i.e., the teleoperation system remains absolutely stable).

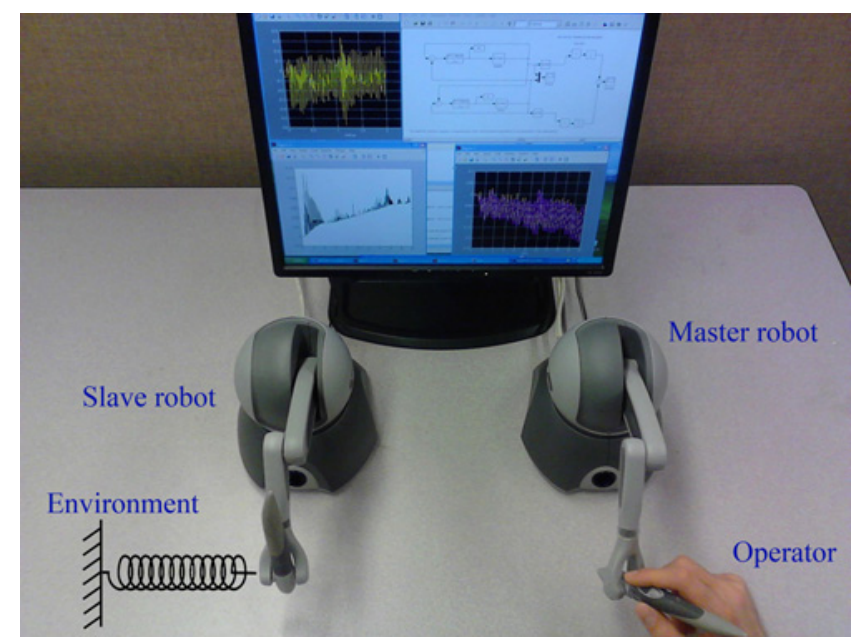

Fig. 7. Experimental setup - The master arm is controlled by the human operator and the slave arm interacts with its environment.

(from Quanser Inc. Markham, ON, Canada). To have accurate sampling times, the simulation runs in the discrete-time and the sampling time of the Simulink is set to the value given for each experiment. The controller(9)has been implemented noting that all of the systems and operations of (9) are in discrete-time.

To be able to verify our absolute stability condition, the mass $m$ and damping $b$ of the robots had to be found through grey-box system identification in a separate experiment. The Omni robots were found to have small but non-negligible joint friction. The viscous friction term was lumped in the damping term and the Coulomb friction term was identified and feedforward compensated in the controller. The values of the mass (inertia, as it corresponds to rotational motion) and the total damping were found to be $m=1.503 \times 10^{-2} \pm 1.7 \times 10^{-4} \mathrm{Kg} \cdot \mathrm{m}^{2}$ and $b=4.624 \times 10^{-2} \pm 1.1 \times 10^{-3} \mathrm{Kg} . \mathrm{m}^{2} / \mathrm{s}$. Also, in a separate experiment, the static model of the nonlinear spring acting as the slave environment was found. The experiment confirmed that the spring was a passive system due to non-negative dissipated energy.

Two series of experiment were conducted to verify the validity and accuracy of the theoretical absolute stability condition (43) for different scaling and controller ratio values. In the first set, it is assumed that the position scaling and the controller ratio are $n_{c}=n_{p}=1$, while in the second set $n_{p}=n_{c}=3$. The absolute stability regions are shown in Fig. 8 and Fig. 9 with the above scaling factors. In each experiment, the controller gains, the sampling time, and the scaling factors are set to constant values and the master is manipulated by the human operator while the slave robot is interacting with the environment. Based on the given set of parameters and condition (43), a particular experiment will be expected from theory to lie in one of the absolute stability or potential instability regions in Fig. 8 and Fig. 9. In practice, as the operator derives the teleoperator, if the master and slave positions become unbounded or indefinitely oscillating, the teleoperation system judged to be unstable such unstable experiments are marked by circles in Fig. 8 and Fig. 9. On the other hand, if the positions remain bounded, the teleoperation system is judged to be stable - such stable experiments are marked by stars in Fig. 8 and Fig. 9. It is expected 
that all of the experimentally-obtained circles must lie in the theoretically-arrived potential instability region. However, the stars may lie in either the absolute stability region or the potential instability region because it is possible to have a potentially unstable teleoperator that, when coupled to certain human operator and environment couplings, results in a stable teleoperation system.

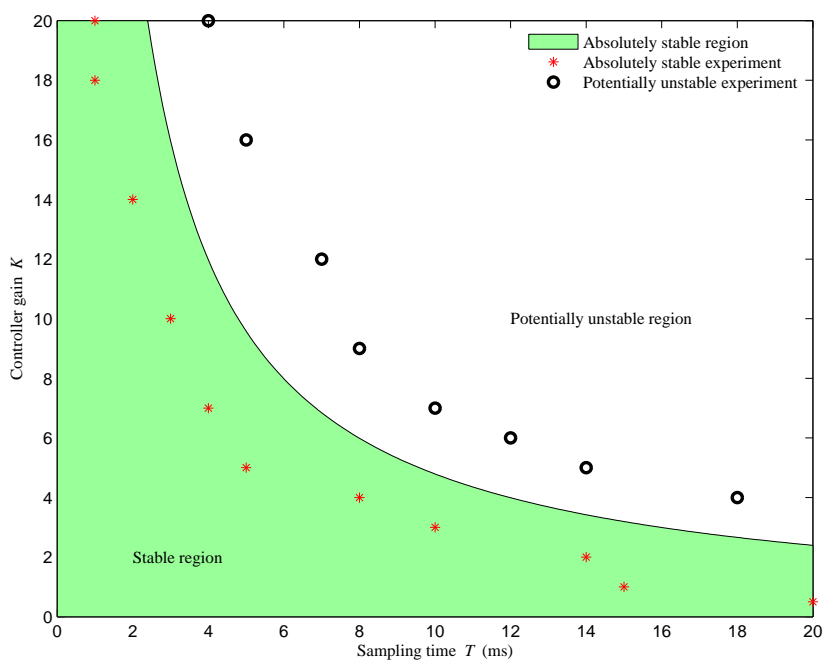

Fig. 8. The theoretical absolute stability region in $K-T$ plane versus experimental data points obtained from a sampled-data teleoperator with $n_{c}=n_{p}=1$.

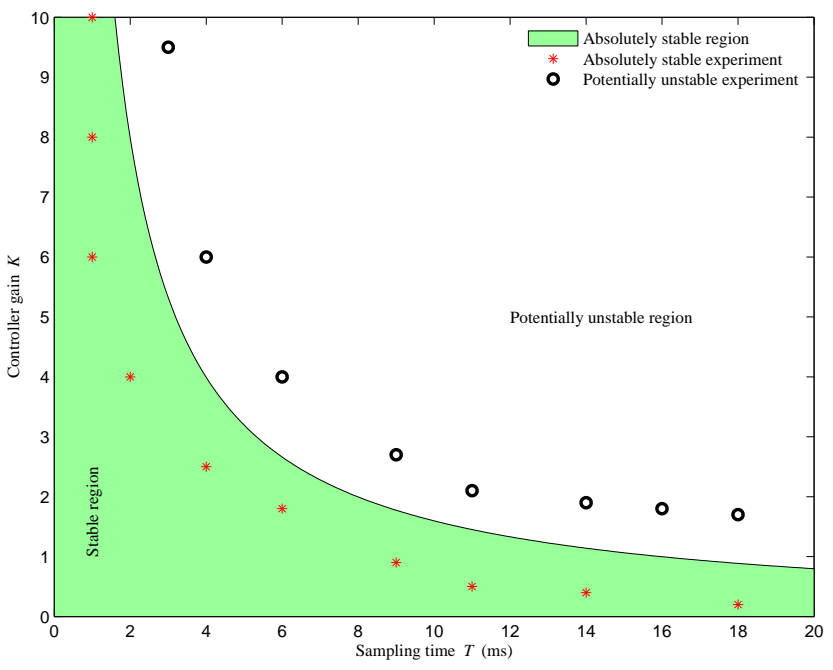

Fig. 9. The theoretical absolute stability region in $K-T$ plane versus experimental data points obtained from a sampled-data teleoperator with $n_{c}=n_{p}=3$.

It was theoretically argued in Remark 1 higher control gains result in a higher transparency of the teleoperation system. In a scaled teleoperation system, transparency requires the ratio of the positions to be $n_{p}$ as described in (10) and, in the context of position error based control, the ratio of the forces to be $n_{c}$ as in (37). Using the sampled-data teleoperation system, various controller gains were experimentally tested with the results shown in Fig. 10. For the fixed sampling time $T=4 \mathrm{~ms}$ and the scaling factors $n_{c}=n_{p}=3$, and $B=0$, the controller gain $K$ changed from 0.5 to 4.5 . In each experiment, the operator moved the master while the slave was in free space. For simplicity of comparison of the position signals in the plots, the slave position has been scaled down by $n_{p}$. The mean square value of the position tracking error has been computed as a measure of the transparency of the teleoperation system. Provided that the sampling time $T$ and the control gain $K$ are in the absolute stability region of Fig. 9, increasing the controller gain decreases the mean square error, confirming that higher controller gains result in higher transparency. In Fig. 10-f, it is seen that the pair of $(T, K)$ happens to be outside of the absolutely stable region of Fig. 9 and, consequently, having a higher controller gain did not result in higher transparency; instead the system became unstable. Thus, while absolute stability puts an upper bound on the control gains, obtaining good transparency will impose lower bounds on them. These emphasize the importance of the tradeoff between the stability and transparency of a teleoperation system. 

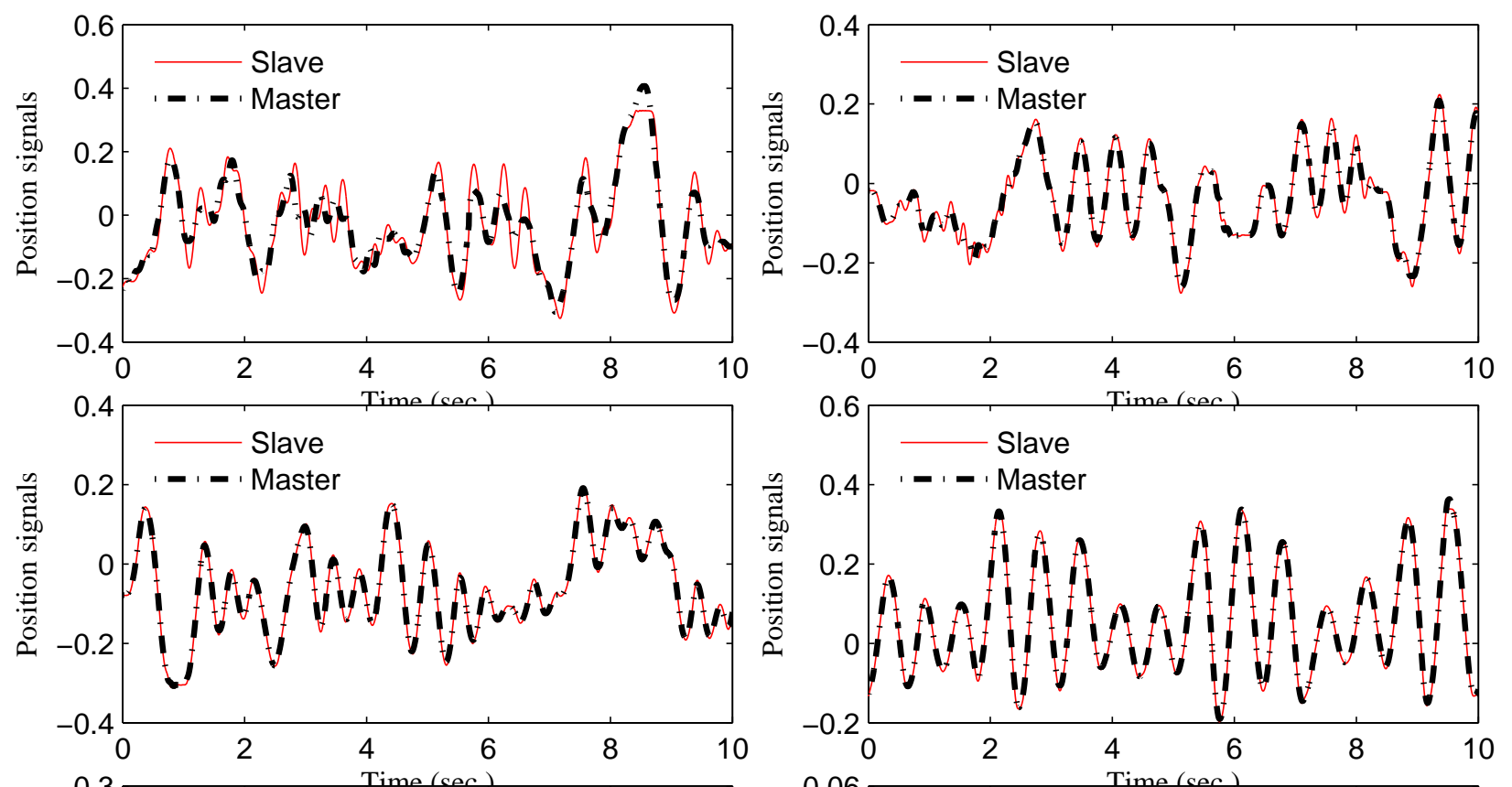

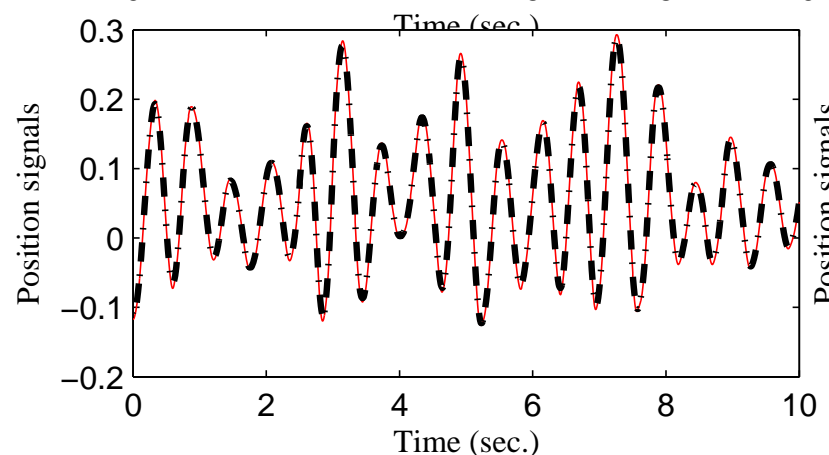

(e)

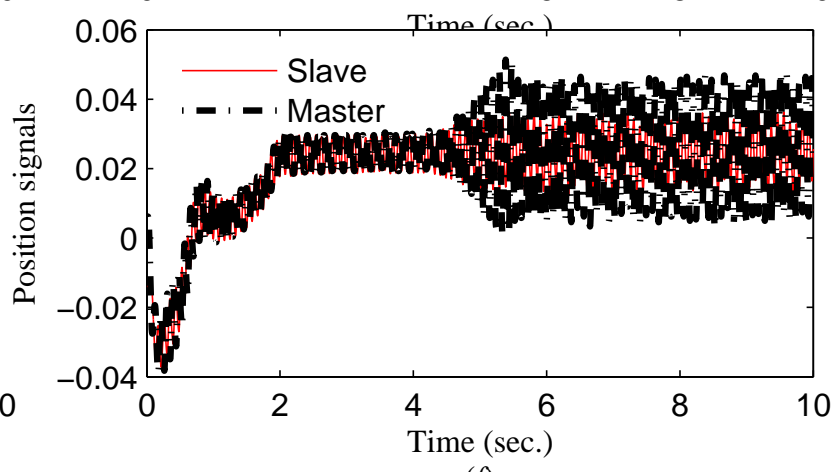

(f)

Fig. 10. The positions of the master and the slave when the operator moved the master and the slave was in free space. The proportional controller gain was $K=0.5, K=1.5, K=2.5, K=3, K=3.5$ and $K=4.5$ for parts (a) to (f), respectively. The means of the tracking errors are $2.20 \times 10^{-3}$, $1.59 \times 10^{-4}, 2.86 \times 10^{-5}, 2.43 \times 10^{-5}, 1.22 \times 10^{-5}$ and $2.59 \times 10^{-4}$ rad, respectively.

In the experimental results of Fig. 11, examples of stable and unstable systems have been demonstrated when the sampling time increased from $2 \mathrm{~ms}$ to $6 \mathrm{~ms}$. In this experiment, the scaling factors were $n_{c}=n_{p}=3$ and the controller gain was $K=4$. While the slave robot is in free space, the operator initially moved the master robot and then released it. As shown in Fig. 11, the positions of the master and the slave converge for $T=2 \mathrm{~ms}$, which satisfies (40), while they oscillate indefinitely after the operator releases the master for $T=6 \mathrm{~ms}$, which violates (40).

\section{Case Study: Effect of Sampling Time on System Absolute Stability and Task Performance in TEleOPERATED FLipPing OF A SWITCH}

To demonstrate the coupling between the absolute stability bound in (40) and the performance of a task carried out through a sampled-data teleoperation system, a task is considered in which a three-way slide switch is flipped by the operator. Trying to accomplish this task with a sampling time of $17 \mathrm{~ms}$ for the discrete-time teleoperation controller, experimental trials showed that the controller gains needed to be higher than the maximum value allowed by the stability bound in (40), which is not allowed. By reducing the sampling time, it is possible to increase the stability margin (i.e., the maximum admissible controller gain obtained from (40) and make it possible for the operator to perform the task successfully. In the following, the details of the task process and the effect of the sampling time on the system absolute stability and the operator task performance will be elaborated.

Fig. 12 shows a three-way slide switch. The objective of the task is to flip the switch using the teleoperation system from state 1 to state 2 but not to state 3 . For the operator to perform the task successfully, the teleoperation controller should ensure that the master/slave position tracking error is less than the position difference between states 2 and 3 of the switch. Such a small position error can only be reached when the teleoperation controller gains are selected sufficiently large (in general, large 


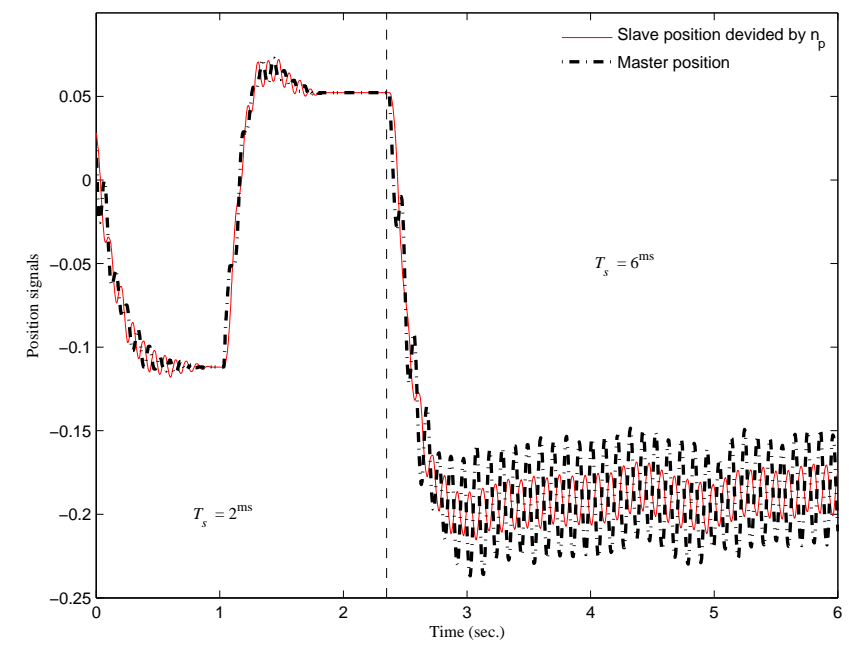

Fig. 11. The position of the master and slave. The sampling time is $T=2^{\mathrm{ms}}$ in $0<t \leq 2.35^{\mathrm{ms}}$ and $T=6^{\mathrm{ms}}$ in $2.35<t<6^{m s}$.

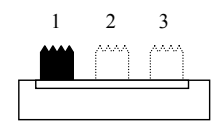

Fig. 12. A three-way slide switch where the objective of the task is to flip it from state 1 to state 2 but not to state 3 .

gains correspond to high teleoperation transparency). Increasing the controller gain, however, jeopardizes the system stability as the sampling time-dependent bound given in (40) indicates. However, by decreasing the sampling time one can always increase the stability margin of the system such that the admissible control gains are large enough to ensure satisfactory task performance. Otherwise, for a given sampling time, it may or may not be possible to achieve the required transparency while maintaining stability.

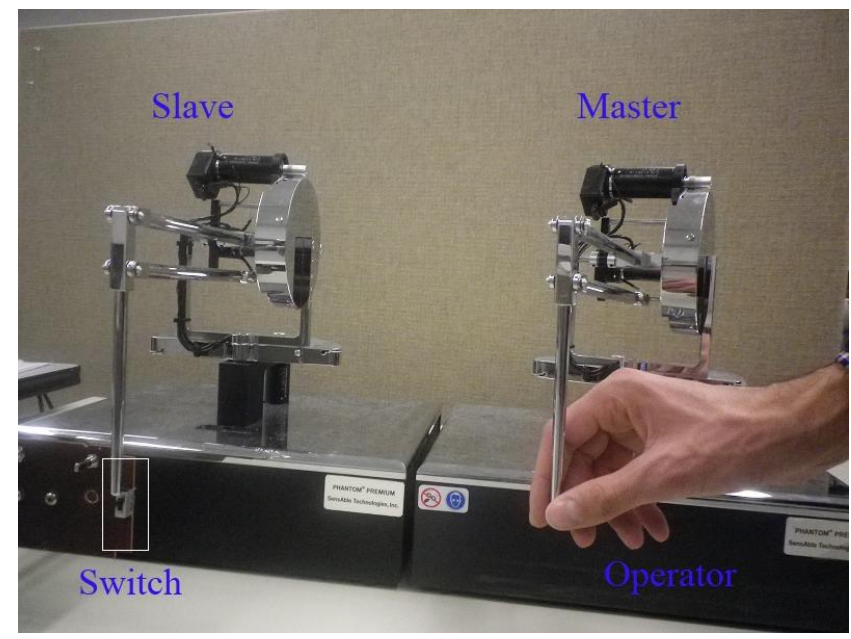

Fig. 13. The experimental setup for flipping the three-way slide switch.

To demonstrate the above, a set of experiments is conducted on a teleoperation system consisting of two Phantom Premium haptic devices (Sensable Technologies, Inc., Wilmington, MA, USA) for flipping the three-way slide switch 13. Initially, the sampling time is set to $17 \mathrm{~ms}$. It is observed that a proportional gain of 3 (for the master and the slave controllers) stabilizes the system but the user cannot successfully teleoperate the switch, failing to flip the switch from state 1 to state 2 without going to state 3. This phenomenon is caused by the relatively low control gain for the slave robot, which results in the accumulation of control actions (forces) until the position error builds up to a large enough threshold at which the slave control action can overcome the switch stiction. Increasing the controller gain from 3 to 5 for the same sampling time makes the teleoperation system unstable; it will be shown that a controller gain of 5 is large enough to ensure good transparency and satisfactory performance of the task, if the sampling time can be lowered. Thus, the only way to achieve the desired transparency is to decrease the sampling time as shown in Fig. 14. 


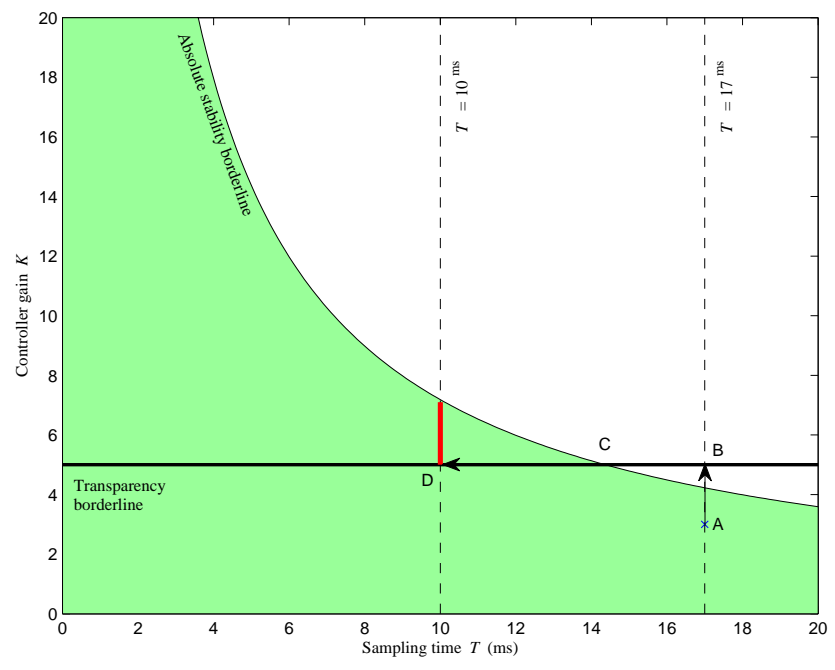

Fig. 14. Transparency and absolute stability of the switching task. Point A corresponds to an absolutely stable but non-transparent teleoperation system, Point B corresponds to an unstable teleoperation system in which the controller gain meets the transparency requirement, Point C corresponds a teleoperation system with the largest sampling time satisfying both transparency and absolute stability conditions, and Point D corresponds to an absolutely stable and transparent teleoperation system

The vertical axis in Fig. 14 shows the controller gain, which has a direct relationship to teleoperation transparency and operator task performance. The horizontal axis is the sampling time of the discrete-time controller. A transparency boundary exists in the form of a nearly horizontal line in this plane, above which the controller gains are high enough to ensure that the operator can correctly flip the switch. The absolute stability boundary of (40) is also shown as a curved line below which the controller gain is low enough to ensure the absolute stability. The dashed vertical line indicates a fixed sampling time, typically upper bounded by hardware limitations in the experimental setup. For our system with the sampling time of 17 ms, the vertical line is at a location where there is no overlap between the transparent and the absolutely stable segments of the dashed vertical line. Consequently, with the given sampling time, there is no choice for the controller gain to be stable and concurrently satisfy the transparency requirement. As the vertical line for the sampling time moves to the left, it reaches a point (at about $14.5 \mathrm{~ms}$ ) where stability and transparency are simultaneously met, corresponding to the maximum allowed sampling time and the minimum allowed gain for the controller (point $\mathrm{C}$ in Fig. 14). As the vertical line moves further to the left, there is a segment of the line that sits below the absolute stability borderline and above the transparency borderline. By moving to smaller sampling times, this segment of the vertical line expands, allowing us to achieve higher transparencies (by going to higher control gains without jeopardizing the stability). The above example illustrates that increasing the transparency while preserving the stability will be made possible by a reduction in the sampling time.

\section{CONCLUSIONS AND Future WORK}

In this paper, a condition for absolute stability of a position error based, sampled-data, scaled teleoperation system has been found using the small gain theorem. The proposed analysis takes into account the exact models of the discretization components such as the zero-order-hold and the sampler. Instead of requiring the passivity of the teleoperator (i.e., the teleoperation system excluding the operator and the environment), absolute stability is merely concerned with the closed-loop stability of the teleoperation system having assumed the passivity of the environment and the human operator, and is less conservative than passivity. Unlike passivity, the absolute stability of a teleoperator allows the teleoperator to be non-passive and involve arbitrary scaling of position and/or force.

The derived absolute stability condition has been simplified for certain controller structures, arriving at bounds on the controller parameters, the sampling time, the master and the slave robot dampings, and the position and force scaling factors. The conditions have been verified in a set of experiments using a dual Phantom Omni teleoperation system. For future work, the condition will be extended to sampled-data scaled teleoperation systems that use the 4-channel control architectures, and when there is time delay in the communication channel between the master and the slave.

\section{REFERENCES}

Abbott, J., Okamura, A., 2003. Analysis of virtual fixture contact stability for telemanipulation. In: Proceedings of IEEE/RSJ International Conference on Intelligent Robots and Systems. Vol. 3. pp. 2699-2706.

Anderson, R., Spong, M., 1989. Bilateral control of teleoperators with time delay. IEEE Transactions on Automatic Control $34(5), 494-501$. 
Arcara, P., Melchiorri, C., 2002. Control schemes for teleoperation with time delay: A comparative study. Robotics and Autonomous Systems (38), 49-64.

Aziminejad, A., Tavakoli, M., Patel, R., Moallem, M., 2008. Transparent time-delayed bilateral teleoperation using wave variables. Control Systems Technology, IEEE Transactions on 16 (3), 548 -555.

Berestesky, P., Chopra, N., Spong, M., 2004. Discrete time passivity in bilateral teleoperation over the Internet. In: Proceedings of International Conference on Robotics and Automation. New Orleans, LA, pp. 4557-4564.

Boukhnifer, M., Ferreira, A., 2006. Wave-based passive control for transparent micro-teleoperation system. Robotics and Autonomous Systems 54 (7), $601-615$.

Cho, H. C., Park, J. H., 2002. Impedance controller design of Internet-based teleoperation using absolute stability concept. In: IEEE/RSJ International Conference on Intelligent Robots and Systems, 2002. Vol. 3. pp. 2256 - 2261 vol.3.

Cho, H. C., Park, J. H., 2005. Stable bilateral teleoperation under a time delay using a robust impedance control. Mechatronics 15 (5), 611-625.

Colgate, J., Schenkel, G., 1997. Passivity of a class of sampled-data systems: Application to haptic interfaces. Journal of Robotic Systems 14 (1), 37-47.

Diolaiti, N., Niemeyer, G., Barbagli, F., J.K. Salisbury, J., 2006. Stability of haptic eendering: discretization, quantization, time delay, and coulomb effects. IEEE Transactions on Robotics 22 (2), 256- 268.

Gil, J., Avello, A., Rubio, A., Florez, J., 2004. Stability analysis of a 1 DOF haptic interface using the routh-hurwitz criterion. IEEE Transactions on Control Systems Technology 12 (4), 583-588.

Gillespie, R., Cutkosky, M., 1996. Stable user-specific rendering of the virtual wall. In: Proceedings of the ASME International Mechanical Engineering Conference and Exposition. Vol. 58. Atlanta, GA, pp. 397-406.

Haddadi, A., Hashtrudi-Zaad, K., 2009. Least conservative robust stability condition for linear bilateral teleoperation control systems. In: EuroHaptics conference, 2009 and Symposium on Haptic Interfaces for Virtual Environment and Teleoperator Systems. World Haptics 2009. Third Joint. pp. $220-225$.

Hannaford, B., 1989. A design framework for teleoperators with kinesthetic feedback. IEEE Transactions on Robotics and Automation 5, 426-434.

Haykin, S., 1970. Active Network Theory. Addison-Wesley, Reading, MA.

Hogan, N., 1989. Controlling impedance at the man/machine interface. In: Proceedings of IEEE International Conference on Robotics and Automation. pp. 1626-1631 vol.3.

Hokayem, P. F., Spong, M. W., 2006. Bilateral teleoperation: An historical survey. Automatica 42 (12), 2035 - 2057.

Jazayeri, A., Tavakoli, M., 2010. Stability analysis of sampled-data teleoperation systems. In: Proceedings of Conference on Decision and Control. Atlanta, GA, pp. 3608-3613.

Jazayeri, A., Tavakoli, M., 2011. A passivity criterion for sampled-data bilateral teleoperation systems. In: Proceedings of World Haptics Conference. Istanbul, Turkey.

Kosuge, K., Itoh, T., Fukuda, T., 1996. Scaled telemanipulation with communication time delay. In: Robotics and Automation, 1996. Proceedings., 1996 IEEE International Conference on. Vol. 3. pp. 2019 -2024 vol.3.

Lawrence, D. A., 1993. Stability and transparency in bilateral teleoperation. IEEE Transactions on Robotics \& Automation 9 , 624-637.

Leung, G., Francis, B., 1992. Bilateral control of teleoperators with time delay through a digital communication channel. In: Proceedings of the Thirtieth Annual Allerton Conference on Communication, Control and Computing. pp. $692-701$.

Mahvash, M., Okamura, A. M., 2007. Enhancing transparency of a position-exchange teleoperator. World Haptics Conference, 470-475.

Matsuoka, Y., Howe, R., 2000. Hand impedance change during learning of a novel contact task. In: World Congress on Medical Physics and Biomedical Engineering.

Mendez, V., Tavakoli, M., 2010. A passivity criterion for n-port multilateral haptic systems. In: Proceedings of Conference on Decision and Control. Atlanta, GA, pp. 3608-3613.

Nuno, E., Basanez, L., Ortega, R., 2011. Passivity-based control for bilateral teleoperation: A tutorial. Automatica 47 (3), 485 $-495$.

Ogata, K., 1995. Discrete-Time Control Systems, 2nd Edition. Prentice Hall.

Ryu, J. H., Kwon, D. S., Hannaford, B., 2004. Stable teleoperation with time-domain passivity control. IEEE Transactions on Robotics and Automation 20 (2), 365-373.

Secchi, C., Stramigioli, S., Fantuzzi, C., 2007. Control of Interactive Robotic Interfaces: A Port-Hamiltonian Approach (Springer Tracts in Advanced Robotics). Springer-Verlag New York, Inc., Secaucus, NJ, USA.

Sheng, J., Liu, P., 2004. A review of bilateral sampled-data control of teleoperators. In: IEEE International Conference on Robotics and Biomimetics, 2004. pp. 385 -390.

Tavakoli, M., Aziminejad, A., Patel, R., Moallem, M., 2008. Discrete-time bilateral teleoperation: modelling and stability analysis. Control Theory and Applications, IET 2 (6), 496-512.

van der Schaft, A., 1999. L2-Gain and Passivity in Nonlinear Control, 2nd Edition. Springer-Verlag New York, Inc., Secaucus, NJ, USA. 


\section{APPENDIX}

The controller defined in (9) is a PEB controller, which is the most commonly used controlled due to the fact that it does not require force measurement. In another controller method, the slave environment contact forces are measured and transmitted to the master side, which is known as direct force reflecting (DFR) control. In the following it will be shown that the absolute stability analysis of the continuous-time teleoperation system with a DFR control is not satisfied and hence the absolute stability of the sampled-data teleoperation system is not satisfied. For a continuous-time bilateral teleoperation system the hybrid matrix for the DFR ( also known as force-position) control reads as

$$
H=\left[\begin{array}{cc}
Z_{m} & 1 \\
-\frac{C_{s}}{Z_{t s}} & \frac{1}{Z_{t s}}
\end{array}\right]
$$

where $Z_{t s}=Z_{s}+C_{s}, Z_{s}$ and $Z_{m}$ are the impedance of the slave robots, and $C_{s}$ is the slave controller. It can be seen that free motion force tracking is achieved $H_{12}=1$ but free-motion impedance which is ideally zero cannot be accomplished $H_{11} \neq 0$. Obviously, the free-motion impedance $H_{11}$ in the DFR case is closer to zero compared to the PEB controller 45 . As it can be seen, for both PEB and DFR controllers the hybrid matrices cannot reach the values of the ideal transparency matrix because of $H_{12}$ and $H_{22}$ values, which emphasizes that for ideal transparency at least three channel of the 4-channel controllers are required.

Theorem 3. The teleoperation system with a DFR controller is absolutely stable if the controller derivative and proportional gains are positive, i.e. $k_{v_{s}}, k_{p_{s}}>0$ and also $\left|C_{s}\right| \gg\left|Z_{s}\right|$.

Proof: Using Llewellyn Theorem the system with hybrid matrix of $H(s)$ is absolutely stable if

1) $H_{11}$ and $H_{11}$ have no poles in the right half plane (RHP).

2) Pure imaginary poles of $H_{11}$ and $H_{22}$ are simple and have positive residue.

3) For all real positive frequencies $\omega$,

$$
\begin{gathered}
\operatorname{Re} H_{11}(j \omega) \geq 0 \\
\operatorname{Re} H_{22}(j \omega) \geq 0 \\
2 \operatorname{Re} H_{11}(j \omega) \operatorname{Re} H_{22}(j \omega) \\
-\operatorname{Re} H_{12}(j \omega) \operatorname{Re} H_{21}(j \omega) \\
-\mid \operatorname{Im} H_{12}(j \omega) \operatorname{Im} H_{21}(j \omega) \quad \geq 0
\end{gathered}
$$

First, $H_{11}$ has no poles and the characteristic polynomial for $H_{22}$ is $M_{s} s^{2}+k_{v_{s}} s+k_{p_{s}}$, which has no RHP poles if $k_{v_{s}}, k_{p_{s}}>0$. Also, $\operatorname{Re} H_{11}=0$ and $\operatorname{Re} H_{22}$ is

$$
\operatorname{Re} H_{22}=\frac{k_{v_{s}}}{k_{v_{s}}^{2}+\left(-k_{p_{s}} / \omega+M_{s} \omega\right)^{2}}
$$

which is non-negative if $k_{v_{s}}>0$. In addition, the third condition in (48) yields

$$
\operatorname{Re}\left(\frac{C_{s}}{C_{s}+Z_{s}}\right)-\left|\frac{C_{s}}{C_{s}+Z_{s}}\right| \geq 0
$$

which is possible only when the controller $C_{s}$ is greater than the impedance of the slave robot $\left|C_{s}\right| \gg\left|Z_{s}\right|$.

Based on the Theorem (3), the continuous-time teleoperation system with DFR controller is absolutely stable only if the controller gains are set to be infinity. For a sampled-data system, since discretization does not make an unstable system stable, the sampled-data equivalent system is not stable. 